\title{
Health Inequality over the Life-Cycle
}

\author{
Timothy J. Halliday ${ }^{* \dagger}$ \\ University of Hawai'i at Mānoa and IZA
}

First Draft: August 10, 2009

June 20, 2011

Working Paper No. 11-7

\begin{abstract}
We consider the covariance structure of health. Agents report their health status on the basis of a latent health stock that is determined by permanent and transitory shocks, and time invariant fixed effects. At age 25, permanent
\end{abstract}

*Address: 2424 Maile Way; Saunders Hall 533; Honolulu, HI 96822. E-Mail: halliday@hawaii.edu. Tele: (808) 956-8615.

$\dagger$ I would like to thank participants at the following conferences: "Understanding Ageing" held at Oxford in April of 2010 and "SES and Health across Generations over the Life-Course" held at the University Michigan in September of 2010. I also would like to thank seminar participants at the University of Hawaii at Mānoa, GRIPS, and Yonsei University. I have benefitted from comments and/or encouragement from Jerome Adda, James Banks, Gabriella Conti, Bo Honoré, Costas Meghir, Robert Michael, and Emmanuel Saez. I am especially indebted to Jim Heckman and to the editor of this journal and two anonymous referees for helping to clarify many points. Finally, I would like to thank PSID and the NIH for funding my travel to Ann Arbor and the Maui High Performance Computing Center which I used briefly. 
shocks account for $5 \%$ to $10 \%$ of the variation in health. At age 60 , this percentage rise to between $60 \%$ and $80 \%$. We document a gradient in which permanent shocks matter less for college-educated people and for women.

JEL Code: I1, C5

Key Words: health, dynamic panel data models, variance decomposition

\section{Introduction}

We consider a model of health evolution in which health shocks can be either permanent or transitory. As individuals age, permanent shocks will accumulate. On the other hand, temporary shocks will affect health for a brief period, but will then dissipate. This view of health as a non-stationary process in which the burden of past events persists until death has its roots in stress models from Epidemiology as discussed by McEwen and Stellar (1993) and Seeman, Singer, Rowe, Horwitz, and McEwen (1997).

However, during the past fifteen years, these models of health have started to penetrate Health Economics. The first instance of this that we are aware of is Deaton and Paxson (1998a). They point out that stress models have the desirable property that they imply that health inequality in the cross-section will increase as 
cohorts age and they provide empirical support for this. In addition, these models bear a nice concordance with Deaton and Paxson (1994) who show a similar result for consumption inequality in a variety of contexts. Notably, that consumption inequality within cohorts should widen with age also happens to be an implication of the Permanent Income Hypothesis. More recently, stress models have formed the basis of the estimations of Adda, Banks, and von Gaudecker (2009) who adopt the permanent-transitory model commonly used in the earnings progression literature (e.g. Abowd and Card (1989) and Meghir and Pistaferri (2004)).

In this paper, we ask ourselves three questions. First, when compared to other factors that impact health status, how important are permanent health shocks? Second, how does the importance of permanent shocks depend on socioeconomic status (SES)? Third, how does the permanent-transitory model of health compare to a simpler alternative with only time-invariant endowments and transitory shocks?

This work contributes to a relatively new literature on the dynamics of health that is rooted in the earnings dynamics literature. ${ }^{1}$ In one of the earliest studies in this literature, Shakotko (1980) investigated the formation of health and cognitive development in early childhood using factor structure models. More recently, studies, such as Adda, Banks, and von Gaudecker (2009), Adams, Hurd, McFadden, Merrill,

\footnotetext{
${ }^{1}$ Notable examples from the latter include Lillard and Willis (1978), Abowd and Card (1989), Baker (1997), Meghir and Pistaferri (2004), and Guvenen (2009).
} 
and Ribeiro (2003), and Borsch-Supan, Heiss, and Hurd (2003), have investigated the joint dynamics of health and income using dynamic panel data techniques. These studies center largely on eliciting the causal pathways between health and SES. Other studies, such as Contoyannis, Jones, and Rice (2004a), Contoyannis, Jones, and Rice (2004b), Halliday (2008), and Carro and Traferri (2010), are more closely tied to the labor economics literature on income and employment dynamics (e.g. Hyslop (1999)). These studies focus exclusively on health status and emphasize the statistical properties of health dynamics by modeling health as a discrete variable and attempting to identify state dependence in the presence of unobserved heterogeneity. However, despite recent progress, this is very much a fledgling field.

We further this field by exploring health inequality from a perspective that has largely been ignored in the literature. Much of the extant literature on health inequality has focused on correlations between health and SES and disentangling causality between the two as in Adda, Banks, and von Gaudecker (2009) and Adams, Hurd, McFadden, Merrill, and Ribeiro (2003) to name two examples. However, the vast majority of the literature does not focus on understanding the structural underpinnings of health inequality. This sentiment is echoed in Deaton and Paxson (1998a) where they note, " that much of the literature on health inequality is not concerned with inequality in years lived, but with the inequalities in health outcomes 
across socioeconomic groups."

To help us to better understand health inequality and its evolution over the life-course, we model health as a continuous latent variable that forms the basis of a survey respondent's self-reported health status (SRHS). Latent health depends on three factors: individual specific endowments, transitory shocks, and permanent shocks. We employ the Panel Study of Income Dynamics (PSID) to estimate the model using a Simulated Method of Moments (SMM) estimator. Given our parameter estimates, we are able to decompose the contribution of the persistent shock vis-à -vis the total variance of latent health.

Our main findings can be summarized as follows. First, we find that, at age 25, permanent shocks roughly account for between $5 \%$ and $10 \%$ of the total variation in health. At age 60, this percentage is between $60 \%$ and $80 \%$ depending on educational attainment and gender. Second, we find that permanent shocks matter less for college-educated people and women. This finding complements Case and Deaton (2005) who conjecture that those with less education will exhibit a steeper decline in health with age because they tend to use their bodies more in their occupations. Second, we compare the stress model of health to a common alternative model, namely, the random effects Probit model. This model is a nested alternative to the stress model in which the variance of the permanent shock is set to zero. We see that 
the performance of the stress model against this alternative decreases monotonically with educational attainment for both women and men. This is consistent with the variance decompositions as it suggests that permanent shocks matter less for more educated people.

The balance of this paper is organized as follows. Next, we set up our econometric model and discuss estimation of its parameters. After that, we discuss our data. We then go on to discuss our findings. Finally, we conclude.

\section{A Stress Model of Health}

We now formalize a stress model of health as discussed in McEwen and Stellar (1993) and Seeman, Singer, Rowe, Horwitz, and McEwen (1997). At the core of these models is a notion termed allostatic load which, to paraphrase, is the cumulative physiologic toll exacted on the body through multiple attempts to cope with stressors. Specifically, Seeman, Singer, Rowe, Horwitz, and McEwen (1997) say that allostatic load can be viewed as, "an index of the relative degree of failure at a physiological level (i.e. a marker of the cumulative, physiologic costs of previous efforts to cope with life's slings and arrows."

To fix ideas, we follow Adda, Banks, and von Gaudecker (2009) and postulate 
the following model for individual $i$ 's latent health at time $t$ (defined as $h_{i t}^{*}$ ):

$$
h_{i t}^{*}=\delta+\gamma_{i}+u_{i t}+\varepsilon_{i t}
$$

There are three key terms in the model: endowments (denoted by $\gamma_{i}$ ), and a permanent and a transitory shock to health (denoted by $u_{i t}$ and $\varepsilon_{i t}$, respectively). The term $\delta$ is a constant. The permanent shock is modeled as a random walk with drift:

$$
u_{i t}=\eta+u_{i(t-1)}+e_{i t}
$$

where the drift term is given by $\eta$. The permanent shock implies that latent health at time $t$ will depend on $\sum_{s=0}^{t-1} e_{i(t-s)}$. This term represents allostatic load in our model. Finally, we assume that the transitory shock follows a white noise process. This is a special case of Adda, Banks, and von Gaudecker (2009) who allow the transitory shock to follow an MA(q). The process is observed until $T$.

We treat the initial condition as follows. We assume that the process begins at $t=1$ and that $u_{i 0}=0$. Similar to Halliday (2008), we assume that the process begins during a person's twenties after their childhood has ended. We assume that the initial condition is age 25 , so that $t=1$ corresponds to age 25 . We do so because 
we suspect that the process guiding a child's health should be substantially different than the process governing the health of an adult. ${ }^{2}$

Each component of equation (1) has an interpretation. The persistent shock, which is modeled as $e_{i t}$, represents events that leave a residue on one's health, such as onset of chronic illness or accidents that have lasting effects. Endowments, modeled as $\gamma_{i}$, represent time-invariant personal characteristics formed early in life that affect a person's health throughout the life-course. The term $\varepsilon_{i t}$, which models transitory shocks, could include mild bouts of illnesses, such as the flu or broken bones.

Stacking the persistent and transitory shocks as $u_{i} \equiv\left(u_{i 1}, \ldots, u_{i T}\right)^{\prime}$ and $\varepsilon_{i} \equiv$ $\left(\varepsilon_{i 1}, \ldots, \varepsilon_{i T}\right)^{\prime}$, the covariance matrix is then

$$
\left(\begin{array}{c}
\gamma_{i} \\
u_{i} \\
\varepsilon_{i}
\end{array}\right) \sim N\left(\mathbf{0},\left[\begin{array}{ccc}
\sigma_{\gamma}^{2} & \mathbf{0}_{1, T} & \mathbf{0}_{1, T} \\
\mathbf{0}_{T, 1} & \boldsymbol{\Sigma}^{P S} & \mathbf{0}_{T, T} \\
\mathbf{0}_{T, 1} & \mathbf{0}_{T, T} & \boldsymbol{\sigma}_{\varepsilon}^{2} \mathbf{I}_{T}
\end{array}\right]\right)
$$

\footnotetext{
${ }^{2}$ One interpretation of $\gamma_{i}$ is that it is the cumulative sum of all investments that took during the agent's childhood.
} 
where

$$
\boldsymbol{\Sigma}^{P S}=\left[\begin{array}{cccc}
\sigma_{e}^{2} & \ldots & \ldots & \sigma_{e}^{2} \\
\vdots & 2 \sigma_{e}^{2} & \ldots & 2 \sigma_{e}^{2} \\
\vdots & \vdots & \ddots & \vdots \\
\sigma_{e}^{2} & 2 \sigma_{e}^{2} & \ldots & T \sigma_{e}^{2}
\end{array}\right]
$$

This structure implies that latent health will be non-stationary, serially correlated at all leads and lags, and highly heteroskedastic. ${ }^{3}$ Finally, the latent variable structure requires a normalization, so we set $\sigma_{\varepsilon}^{2}=1 .^{4}$

We can decompose the variance of the latent health variable as follows. Given our assumptions on the initial condition, we can write

$$
h_{i t}^{*}=\delta+\gamma_{i}+\eta t+\varepsilon_{i t}+\sum_{s=0}^{t-1} e_{i(t-s)}
$$

\footnotetext{
${ }^{3}$ While writing this paper, we also attempted to estimate some more general models. One generalized the white noise process for the transitory shock to an MA(1) process. The other also allowed the permanent shock to be correlated with the endowments. When we optimized the SMM objective function from separate starting values, we found that the two resulting minimized objective functions were numerically very close, but the MA and correlation coefficients that optimized the functions were drastically different. This is an identification problem. Consequently, we decided not to explore these models in this paper.

${ }^{4}$ Identification of $\theta$ works as follows. The error structure in our model has a one-factor representation and so the linear index can be written as $\kappa_{t} \alpha_{i}+v_{i t}$ where the $v_{i t}$ are serially uncorrelated. Subject to a normalization, the parameters in this model are easily identified. These parameters can then be mapped into $\theta$. In other words, $\theta$ can be backed out from the parameters from the one-factor model. For a more detailed treatment, we refer the reader to Heckman (1981). Finally, note that the one-factor representation imposes non-stationarity and so precludes stationary AR processes. This weak identification problem was not an issue for the simpler model that we estimated as different starting values resulted in the same parameter estimates.
} 
which, in turn, implies

$$
\sigma_{h_{i t}^{*}}^{2}=\sigma_{\gamma}^{2}+t \sigma_{e}^{2}+1
$$

At any point in time, health inequality depends on the variances of the endowments and both types of shocks.

This formula has several important implications. First, it tells us that inequality in latent health increases as the cohort ages. This result is consistent with empirical evidence both on health inequality (Deaton and Paxson (1998a) and Deaton and Paxson (1998b)) and consumption inequality (Deaton and Paxson (1994) and Primiceri and van Rens (2009)). Second, permanent shocks explain an increasing portion of health inequality within a cohort. Hence, as people age and adverse health events accumulate for some but not others, the disparity between the healthiest and unhealthiest will widen as the cohorts ages.

The econometrician does not observe the individual's latent health stock. Rather, she observes the agent's SRHS, which we denote by $h_{i t}$. The agent's SRHS is reported according to the rule

$$
h_{i t}=d \Leftrightarrow \alpha_{d-1} \geq h_{i t}^{*}>\alpha_{d}
$$

for $d \in\{1, \ldots, 4\}$, where $\alpha_{0}=\infty, \alpha_{1}=0$ and $a_{4}=-\infty$. The health states in relation (6) correspond to the different categorizations of SRHS. Once we account 
for the cut points, the parameter vector to be estimated is $\theta \equiv\left(\delta, \sigma_{\gamma}^{2}, \sigma_{e}^{2}, \eta, \alpha_{2}, \alpha_{3}\right) .^{5}$

At this point, we clarify three issues. First, there is an inverse relationship between $h_{i t}$ and $h_{i t}^{*}$, so that higher values of $h_{i t}^{*}$ and lower values of $h_{i t}$ denote better health. Our reason for doing this is that SRHS measures are such that lower values correspond better health, but when health is incorporated in economic models as a continuous variable (most notably by Grossman (1972)), higher values correspond to better health. Hence, by doing this we maintained consistency with both the standard way of measuring SRHS and also conventions in the literature on health investment. Second, there are only three finite cut points because we consolidated the "fair" and "poor" states into a single category. We do this because there were often too few observations of poor health in certain age cells which created difficulties pinning down the bottom cut point with all five categories. Third, as in Carro and Traferri (2010), we normalized $\alpha_{1}$ to zero because we included a constant in equation (1).

\footnotetext{
${ }^{5}$ An important issue when working with SRHS data is cut-point shifting in which certain groups report systematically higher or lower health than other groups (see Lindeboom and van Doorslaer (2004), for example). Fully addressing this issue is beyond the scope of this paper, but we do partially address it in the following way. Consider a binary model with heterogeneity in the constant and cut-point:

$$
h_{i, t}=1\left(\alpha_{i}+\varepsilon_{i, t}>c_{i}\right) .
$$

Clearly, the distributions of $\alpha_{i}$ and $c_{i}$ are not separately identified and, so in this simple model, heterogeneity in the constant accounts for both individual differences in latent health and cut-points. A similar argument can be made in our model.
} 


\section{Estimation}

We propose a tractable SMM procedure that matches the probabilities of simulated sequences of $h_{i t}$ with their counterparts in the data. First, we simulate the model and compute the probabilities of appropriate sequences. We collect all of these probabilities in the vector $P^{M}(\theta)$. Next, we collect the analogues of $P^{M}(\theta)$ from the data in $P^{D}$. These probabilities are estimated non-parametrically. Our moments are then defined as $m(\theta) \equiv P^{M}(\theta)-P^{D}$. For reasons documented in Altonji and Segal (1996), we follow Meghir and Pistaferri (2004) and employ Equally Weighted Minimum Distance, which minimizes the objective function:

$$
Q(\theta)=m(\theta)^{\prime} m(\theta)
$$

Once again following Meghir and Pistaferri (2004), we use the block bootstrap in which we re-sample individuals (not individual-time observations) to compute standard errors. ${ }^{6}$ This procedure accounts for correlations in observations within individuals but across time. Further details about the optimization routines and the specific moments that were used in the estimation procedure can be found in the Appendix.

\footnotetext{
${ }^{6}$ We re-sampled the data 50 times.
} 


\section{Data}

We use a sample of Caucasian women and men ages 25 to 60 from PSID waves 1984

to 1997 . We do not use data before 1984 because there is no information on SRHS

prior to this year. We did not go beyond 1997 as the PSID was collected every other

year beyond 1997.

Our main health measure is SRHS, a categorical variable by which the respondent classifies their health into one of five categories: Excellent (SRHS equal to 1), Very Good (SRHS equal to 2), Good (SRHS equal to 3), Fair (SRHS equal to 4), and Poor (SRHS equal to 5). For the main analysis, we also use data on age and educational attainment. Descriptive statistics are reported in Table 1. A detailed discussion of our sample selection is provided in the Appendix. ${ }^{7}$

\footnotetext{
${ }^{7}$ We defend our use of SRHS measures as follows. First, we are ultimately interested in the dynamics of a latent health index that, in turn, determines a person's assessment of their own health. Not only is this exercise of interest in their own right, but it also has potentially important implications for incorporating continuous health measures into structural models of lifecycle behavior. Many longitudinal or repeated cross-sectional data sets contain SRHS, so the methods discussed in this paper can be cheaply implemented by structural modelers who have access to panels or repeated cross-sections of SRHS. Second, it has long been documented that these measures of health correlate well with more objective health measures. Third, many alternative health measures are not without flaws. For example, Baker, Stabile, and Deri (2004) investigated the possibility of measurement errors in self-reported, objective measures of health (such as those from the Health and Retirement Survey) by comparing them with medical records. They concluded that these measurement errors were often quite large and regrettably correlated with labor market activity.
} 


\section{Empirical Results}

We estimate the model for six demographic sub-groups corresponding to three educational categories separated by gender. The first educational category is "College" and corresponds to people who hold college degrees. The second is "HS Grad" and corresponds to people who have more than twelve years of schooling but no college degree. The third is "HS" and corresponds to people with twelve years of schooling or less. This partition of the data closely mimics Meghir and Pistaferri (2004). We report point estimates and their bootstrapped standard errors for these six demographic groups in Table 2.

The two most salient patterns that emerge from this table are that permanent shocks matter less for people with college degrees and for women. For men, we see that the point estimate of $\sigma_{e}^{2}$ is 0.2374 for the College group but 0.3407 and 0.3063 for the HS Grad and HS groups, respectively. For women, we see a similar pattern: 0.1320 for College, 0.2591 for HS Grad, and 0.2916 for HS. In contrast to men, the pattern for women is monotonic in educational attainment. A similar pattern exists across genders. The estimates of $\sigma_{e}^{2}$ are systematically higher for men within educational groups.

The standard errors of $\sigma_{e}^{2}$ also tell a similar story. For the College group, they are 0.1400 and 0.1594 for men and women, respectively. For the HS Grad group, they 
are 0.1013 and 0.1007 . For the HS group, they are 0.0703 and 0.0628 . The standard errors are at least $40 \%$ larger for the College group than for the other groups. This is not merely a function of small sample sizes in the College group as the HS Grad group has smaller samples but lower standard errors. Rather, it reflects that the permanent shocks matter less for the most educated. It also suggests that, for the college-educated, a model with permanent shocks would not perform substantially better than a simple alternative with only a random effect and a transitory shock.

To this end, we computed something akin to a likelihood ratio statistic. To do this, we re-estimated the model subject to the restriction that $\sigma_{e}^{2}=0$ using the same moments and simulations. ${ }^{8}$ We reported the value of the GMM objective function with this restriction as $Q_{\sigma_{e}^{2}=0}(\widehat{\theta})$. We then compared this to the objective function from the unrestricted model which we call $Q_{U N R E}(\widehat{\theta})$. The statistic that we report is $\frac{Q_{U N R E}(\widehat{\theta})}{Q_{\sigma_{e}^{2}=0}(\widehat{\theta})} \cdot{ }^{9}$

The same pattern can be seen looking at these statistics. For college-educated

\footnotetext{
${ }^{8}$ See the Appendix for details on how we drew the simulations.

${ }^{9}$ While the "Holy Trinity" of likelihood based tests can be extended to GMM estimators as discussed in Newey and McFadden (1994), they cannot be applied in our case. The reason is that for the asymptotic theory of these tests to be applicable the parameter value under the null cannot be at the edge of a compact set. If it is, one cannot apply simple asymptotic results such as the Central Limit Theorem to a first-order Taylor expansion. One way to rectify this would be to use a model selection criterion such as the AIC or BIC. However, these will not work either as these methods are likelihood based.
} 
men, we see that the objective function for the unrestricted model is $88.5 \%$ lower than for the restricted model. Moving to the other two educational groups, we see that the statistics are $72.3 \%$ (HS Grad) and $67.8 \%$ (HS). For women, the statistics are $99.7 \%$ (College), $83.1 \%$ (HS Grad), and $69.0 \%$ (HS). For both genders, the statistics increase monotonically with educational attainment consistent with the notion that permanent shocks matter less for more educated people. Similarly, we also see that, within educational groups, the statistics are higher for women than for men suggesting that permanent shocks matter more for men.

We conclude this section by conducting a simple variance decomposition exercise. In Figures 1 through 6, we plot the contributions to the total variance of latent health that can be attributed to the permanent shocks, the endowments, and the transitory shocks. Early in the life-cycle, permanent shocks matter relatively little; their contribution to the overall variance at age 25 is typically under $10 \%$. As people age, however, they become more important. By the mid-thirties, they are the largest constituent of the overall variance of health. By age 60, they constitute between $60 \%$ to $80 \%$ of the variance of health.

The gradients in gender and education that we spoke off can also be seen in these figures, although the education gradient is not terribly pronounced for men. To better see this, we present Table 3 where we report the contributions of the 
permanent shocks at ages 25 and 60 for all six sub-groups. As can seen, permanent shocks matter the least for the college-educated and, for women, there is a monotonic relationship with education. We also see a marked difference in the importance of permanent shocks across genders within educational groups.

\section{Discussion}

The key result of this paper, namely that permanent shocks matter less for more educated people and for women, is consistent with a paper by Case and Deaton (2005). They provide evidence that people who engage in more physical labor will tend to see a steeper decline of health with age. Because of the nature of their work, permanent shocks to health, such as on-the-job injuries or wearing down of cartilage and joints, will matter more for these people. Our results are broadly in line with their paper since the less educated have a higher tendency to be in physically strenuous occupations. A similar argument can also be made for the observed differences across gender.

An important avenue for future work is to compare the simple stress model of health dynamics in this paper to some important alternative models. In particular, researchers should explore how extending the model in this paper to account for 
higher order MA transitory shocks and correlations between permanent shocks and endowments would improve the fit of the model. However, as we discussed in this paper, we attempted to pursue this but we encountered problems with weak identification. Perhaps, panels with larger sample sizes could remedy this.

Researchers may also explore a stationary AR model with heterogeneous trends which is a common alternative to the permanent-transitory model in the earnings dynamics literature (e.g. Baker (1997) and Guvenen (2009)). However, this exercise, while very important, would entail some challenges. In linear models, autocovariances of earnings growth yield a clean way of differentiating between the two models since the permanent-transitory model implies that these autocovariances should go to zero at long lags, whereas the other model does not. Unfortunately, we have a latent variable framework in this paper and, so we cannot do this. Because of this, we would need to rely on non-nested hypothesis testing which may have poor finite sample performance given our data.

However, there are two other findings in the literature that challenge the validity of the AR model with heterogeneous trends First, in Halliday (2008), we explored a model with heterogeneous age trends and state dependence. The data rejected the presence of heterogeneous trends in this related model. Nevertheless, researchers may want to see if replacing state dependence with stationary AR residuals changes 
this. Second, Deaton and Paxson (1998a) and Deaton and Paxson (1998b) showed that there was a tendency for within cohort inequality in numerous health measures to fan out as the cohort aged. In the absence of heterogeneous trends, this finding would necessitate permanent shocks.

Understanding which of these models is more appropriate is important as they have very different implications for consumer behavior. To illustrate, we refer to Deaton (1992), who discusses the implications that different income processes have on consumption behavior in a stripped down life-cycle model with quadratic preferences and a discount factor set to the inverse of the real rate of return. He shows that modeling income either as trend stationary or as difference stationary has radically different implications for consumer behavior. The former (which many believe to be less plausible) implies that consumption is smoother than income, whereas the latter implies the opposite. Analogously, many health economists who have investigated the dynamics of health, have essentially modelled health as trend stationary (e.g. Contoyannis, Jones, and Rice (2004a), Contoyannis, Jones, and Rice (2004b), Halliday (2008), and Carro and Traferri (2010)). We contend that more work is needed using different estimators, modeling assumptions, and data sources if we wish to get a better handle on the stochastic process governing health. As illustrated by Deaton, the consequences of different health processes for consumer behavior may 
be non-trivial. ${ }^{10}$

Finally, our investigation into the volatility of health suggests another dimension of the association between health and SES. As pointed out by Blundell and Preston (1998), "risk averse households with more uncertain incomes than others need to be considered worse off." For example, if there is a precautionary savings motive, then this will result in lower consumption expenditures. A similar argument can be made for the volatility of health. First, to the extent that health shocks impact income, then more volatile health implies more volatile income. Second, since health also has consumption value, then a mean preserving spread in health will lower expected utility for risk averse people. So, our paper suggests that college educated people are better off not only because their health is better but because it is less uncertain. This is another dimension of the gradient that has yet to be mentioned in the literature.

\section{Appendix: Sample Selection}

We first extracted all individuals from the 1984 to 1997 waves of the PSID who were either heads of household or the spouse of a household head. The initial sample size was 24,167 individuals. Next, we dropped people with incomplete health data

\footnotetext{
${ }^{10}$ For example, if health is modelled as an exogenous and continuous variable (as in this paper) and is allowed to impact income, the model is essentially the same as in Deaton (1992).
} 
which brought the sample size to 24,054. After this, we kept only Caucasians resulting in sample size of 14,783 . We then dropped people who were not in the panel continuously. This lowered the sample size to 13,805 . Next, we kept only people between ages 25 and 60, inclusive. This brought the sample size to 11,018. Sample sizes by demographic group are reported in Table 4 . Note that there were an additional 207 people who were missing educational information and, so if we add them to the six samples sizes from the table we obtain 11,018. Finally, as in Meghir and Pistaferri (2004), we included the Survey of Economic Opportunity.

\section{Appendix: Estimation Details}

Optimization To obtain our parameter estimates, we optimized the GMM

objective function using simulated annealing (SA). For each demographic sub-group, we ran the procedure once and then we used the maximizer of that procedure as the starting value for a second run of SA. After this, to be certain that we had the true maximand, we used the maximizer of the second run of SA as the starting value of a final run of Nelder-Mead (NM). The primary advantage of SA over NM is that it is able to go both uphill and downhill which makes it less vulnerable to getting stuck in local minima. The cost is that it is substantially slower than NM. Each run of this 
entire routine (i.e. two runs of SA followed by one run of NM) took approximately 36 hours. Finally, to be entirely sure that we did not have any issues with local minima or weak identification, we ran this whole routine twice using two different starting values. In total, it took 72 hours of computation to obtain the parameter estimates for each demographic sub-group. We did not encounter any problems with local minima or weak identification.

Moments We used two sets of moments in the estimation. The first set contains the unconditional probabilities of the four health states that we consider (i.e. excellent, very good, good, fair/poor). Here, we compute the unconditional probability of these four health states (i.e. excellent, very good, good, fair/poor) for a total of 36 ages (i.e. ages 25 to 60 ). This yields a total of $3 \times 36=108$ moments. These moments are informative of the drift parameter, constant and the cuts. In addition, as shown in Heckman (1981), they are also informative of the variance of the permanent shock. The second set of moments that we used were sequences of length four of health states. We used the three most common sequences for each demographic sub-group at ages $25,30,35,40,45,50,55$, and 60 . The sequences that we used can be found in Tables 5 and $6 .{ }^{11}$ This yields a total of $3 \times 7=21$ additional

\footnotetext{
${ }^{11}$ The table reports the three most common sequences at each age. In the reported fraction, the numerator is the number of occurances of each sequence and the denomitator is the number of people who are present at that age and who remain for at least four periods.
} 
moments. These moments added additional information about the variance of the endowments and permanent shocks. In total, we used 129 moments.

Simulations We simulated 250,000 individuals for each estimation. Because

we had between 1000 and 3000 individuals in each of our demographic sub-groups, this corresponded to between 250 and 85 simulations per individual. We used the same simulations for all of our estimations. This ensured that differences across estimations were due entirely to differences across demographic sub-groups or to different models.

\section{References}

Abowd, J., And D. CARD (1989): "On the Covariance Structure of Earnings and Hours Changes," Econometrica, 57(2), 411-45.

Adams, H., M. Hurd, D. McFadden, A. Merrill, and T. Ribeiro (2003): "Healthy, Wealthy and Wise? Tests for Direct Causal Paths between Health and Socioeconomic Status," Journal of Econometrics, 112(1), 3-56. 
Adda, J., J. Banks, and H. von Gaudecker (2009): "The Impact of Income Shocks on Health: Evidence from Cohort Data," Journal of the European Economics Association, 7(6), 1361-99.

Altonji, J., And L. Segal (1996): "Small-Sample Bias in GMM Estimation of Covariance Structures," Journal of Business and Economic Statistics, 14(3), 353366.

BAKER, M. (1997): "Growth-Rate Heterogeneity and the Covariance Structure of Life-Cycle Earnings," Journal of Labor Economics, 15(2), 338-75.

Baker, M., M. Stabile, and C. Deri (2004): "What Do Self-Reported, Objective Measures of Health Measure," Journal of Human Resources, 39(4), 1067-93.

Blundell, R., and I. Preston (1998): "Consumption Inequality and Income Uncertainty," Quarterly Journal of Economics, 113(2), 603-640.

Borsch-Supan, A., F. Heiss, and M. Hurd (2003): "Healthy, Wealthy, and Knowing Where to Live: Predicted Trajectories of Health, Wealth, and Living Arrangements among the Oldest Old," NBER Working Paper.

Carro, J., and A. Traferri (2010): "Correcting the Bias in the Estimation of a Dynamic Ordered Probit with Fixed Effects of Self-Assessed Health," Unpublished Mimeo. 
Case, A., and A. Deaton (2005): "Broken Down by Work and Sex: How Our Health Declines," in Analyses in the Economics of Aging, ed. by D. Wise. University of Chicago Press, Chicago, IL.

Contoyannis, P., A. Jones, and N. Rice (2004a): "The Dynamics of Health in the British Panel Survey," Journal of Applied Econometrics, 19(4), 473-503.

— (2004b): "Simulation-Based Inference in Dynamic Panel Probit Models: An Application to Health," Empirical Economics, 29(1), 49-77.

Deaton, A. (1992): Understanding Consumption. Oxford University Press, Oxford.

Deaton, A., And C. PaXson (1994): "Intertemporal Choice and Inequality," Journal of Political Economy, 102(3), 437-67.

_ (1998a): "Aging and Inequality in Income and Health," American Economic Review: Papers and Proceedings, 88(2), 248-253.

— (1998b): "Health, Income and Inequality over the Life-Cycle," in Fronteirs in the Economics of Aging, ed. by D. Wise. University of Chicago Press, Chicago, IL.

Grossman, M. (1972): "On the Concept of Health Capital and the Demand for Health," Journal of Political Economy, 80(2), 223-55. 
Guvenen, F. (2009): "An Empirical Investigation of Labor Income Processes," Review of Economic Dynamics, 12(1), 58-79.

Halliday, T. (2008): "Heterogeneity, State Dependence, and Health," Econometrics Journal, 11(3), 499-516.

Heckman, J. (1981): "Statistical Models for Discrete Panel Data," in A Structural Analysis of Discrete Data with Econometric Applications, ed. by D. McFadden, and C. Manski. MIT Press, Cambridge, MA.

Hyslop, D. (1999): "State Dependence, Serial Correlation and Heterogeneity in Intertemporal Labor Force Participation," Econometrica, 67(6), 1255-94.

Lillard, L., And R. Willis (1978): "Dynamic Aspects of Earnings Mobility," Econometrica, 46(5), 985-1012.

Lindeboom, M., and E. van Doorslaer (2004): "Cut-Point Shift and Index Shift in Self-Reported Health," Journal of Health Economics, 23(6), 1083-99.

McEwen, B., and E. Stellar (1993): "Stress and the Individual: Mechanisms Leading to Disease," Archives of Internal Medicine, 153(18), 2093-101.

Meghir, C., and L. Pistaferri (2004): "Income Variance Dynamics and Heterogeneity," Econometrica, 72(1), 1-32. 
Newey, W., and D. McFadden (1994): "Large Sample Estimation and Hypothesis Testing," in Handbook of Econometrics, Volume IV, ed. by R. F. Engle, and D. L. McFadden. North-Holland, Amsterdam.

Primiceri, G., and T. van Rens (2009): "Heterogeneous Life-Cycle Profiles, Income Risk and Consumption Inequality," Journal of Monetary Economics, 56(1), $20-39$.

Seeman, T., B. Singer, J. Rowe, R. Horwitz, and B. McEwen (1997): "Price of Adaptation - Allostatic Load and Its Health Consequences," Archives of Internal Medicine, 157(19), 2259-2268.

Shaкотко, R. (1980): "Dynamic Aspects of Children's Health, Intellectual Development, and Family Economic Status," NBER Working Paper. 
Table 1: Descriptive Statistics

\begin{tabular}{lcc} 
& Men & Women \\
\hline \hline SRHS & 2.15 & 2.26 \\
& $(1.01)$ & $(1.01)$ \\
Years of Education & 13.17 & 12.90 \\
& $(2.71)$ & $(2.62)$ \\
Years of Education $>12$ & 0.53 & 0.49 \\
& $(0.50)$ & $(0.50)$ \\
College Degree & 0.33 & 0.28 \\
& $(0.47)$ & $(0.45)$ \\
Age & 39.39 & 39.55 \\
$\mathrm{~N}$ & $(9.34)$ & $(9.52)$ \\
\hline \hline
\end{tabular}

Reports means and standard deviations in parentheses. 
Table 2: Parameter Estimates College

\begin{tabular}{lcccccc}
\hline \hline & $(1)$ & $(2)$ & $(3)$ & $(4)$ & $(5)$ & $(6)$ \\
\hline \hline$\alpha_{2}$ & -2.3643 & -2.3147 & -2.5106 & -2.2275 & -2.4251 & -2.3087 \\
& $(0.1694)$ & $(0.1962)$ & $(0.2517)$ & $(0.2486)$ & $(0.2128)$ & $(0.1464)$ \\
$\alpha_{3}$ & -4.7677 & -4.4209 & -4.8669 & -4.5117 & -4.9349 & -4.7276 \\
& $(0.4238)$ & $(0.4995)$ & $(0.5333)$ & $(0.4787)$ & $(0.3742)$ & $(0.2990)$ \\
$\delta$ & 0.1205 & -0.2442 & -0.2921 & -0.7033 & -1.0649 & -1.3534 \\
& $(0.1453)$ & $(0.1636)$ & $(0.2142)$ & $(0.2114)$ & $(0.1908)$ & $(0.1736)$ \\
$\eta$ & -0.0367 & -0.0343 & -0.0688 & -0.0402 & -0.0664 & -0.0540 \\
& $(0.0066)$ & $(0.0080)$ & $(0.0148)$ & $(0.0117)$ & $(0.0099)$ & $(0.0089)$ \\
$\sigma_{e}^{2}$ & 0.2374 & 0.1320 & 0.3407 & 0.2591 & 0.3063 & 0.2916 \\
& $(0.1400)$ & $(0.1594)$ & $(0.1013)$ & $(0.1007)$ & $(0.0703)$ & $(0.0628)$ \\
$\sigma_{\gamma}^{2}$ & 1.8518 & 1.8276 & 1.7137 & 1.7417 & 2.0237 & 1.8035 \\
$Q_{U N R E}(\widehat{\theta})$ & $0.2131)$ & $(0.2451)$ & $(0.2824)$ & $(0.2483)$ & $(0.2267)$ & $(0.1666)$ \\
$Q_{\sigma_{e}^{2}=0}(\widehat{\theta})$ & 0.0615 & 0.1089 & 0.1155 & 0.1093 & 0.0514 & 0.0544 \\
$Q_{U N R E}(\widehat{\theta})$ & 0.0695 & 0.1092 & 0.1598 & 0.1316 & 0.0756 & 0.0788 \\
$Q_{\sigma_{e}^{2}=0}(\widehat{\theta})$ & $88.5 \%$ & $99.7 \%$ & $72.3 \%$ & $83.1 \%$ & $67.8 \%$ & $69.0 \%$ \\
Gender & Men & Women & Men & Women & Men & Women \\
$N$ & 1588 & 1321 & 1091 & 1040 & 2870 & 2901 \\
\hline \hline
\end{tabular}

For each parameter, we report the point-estimate and the bootstrapped standard error in parentheses. The value for $Q_{U N R E}(\widehat{\theta})$ corresponds to the objective function maximized subject to no restrictions. The value for $Q_{\sigma_{e}^{2}=0}(\widehat{\theta})$ corresponds to the objective function maximized subject to the restriction that $\sigma_{e}^{2}=0$. 
Table 3: Percentage of Variance Due to Permanent Shocks Age 25

Age 60

\begin{tabular}{lcccc}
\hline \hline & Men & Women & Men & Women \\
\hline \hline College & 0.0768 & 0.0446 & 0.7498 & 0.6269 \\
HS Grad & 0.1115 & 0.0863 & 0.8188 & 0.7728 \\
HS & 0.0920 & 0.0942 & 0.7848 & 0.7892
\end{tabular}


Table 4: Sample Sizes

\begin{tabular}{l||ll} 
& White Men & White Women \\
\hline \hline College & 1588 & 1321 \\
HS Grad & 1091 & 1040 \\
HS & 2870 & 2901
\end{tabular}


Table 5: Sequences, Men

College

HS Grad

HS

\begin{tabular}{|c|c|c|c|c|c|}
\hline \multicolumn{6}{|c|}{ Age 25} \\
\hline 1111 & $=0.254$ & 111 & $\frac{28}{156}=$ & 2222 & .139 \\
\hline & & 2222 & $\frac{18}{156}$ & 1111 & \\
\hline 211 & $\frac{11}{185}=$ & 3222 & $\begin{array}{l}\frac{8}{156} \\
\text { Age : }\end{array}$ & 3333 & \\
\hline 1111 & $\frac{81}{343}=0.236$ & 1111 & & 2222 & $\frac{71}{532}=0.133$ \\
\hline 2 & $\frac{31}{343}=$ & 2222 & $\frac{31}{240}$ & 1111 & \\
\hline 1112 & $\frac{18}{343}=$ & 2221 & $\begin{array}{l}\frac{9}{240} \\
\text { Age }\end{array}$ & 3333 & .051 \\
\hline 1111 & $\frac{99}{406}=0.244$ & 2222 & & 2222 & \\
\hline & $\frac{45}{406}=$ & 1111 & & 3333 & \\
\hline 1112 & $\frac{16}{406}=$ & 3333 & $\begin{array}{l}\frac{10}{242} \\
\mathrm{Age}\end{array}$ & 1111 & \\
\hline 1111 & $\frac{98}{404}=$ & 1111 & & 2222 & \\
\hline & $\frac{38}{404}=$ & 2222 & & 1111 & \\
\hline & $\frac{18}{404}=$ & 3333 & $\begin{array}{l}\frac{12}{206}= \\
\text { Age } 4\end{array}$ & 3333 & \\
\hline 1111 & $\frac{71}{305}=$ & 2222 & $\frac{14}{115}$ & 3333 & \\
\hline & & & & 4444 & \\
\hline 2232 & $\frac{10}{305}=0.033$ & 1111 & $\begin{array}{l}\frac{9}{115}=0.078 \\
\text { Age } 50\end{array}$ & 2222 & 0.084 \\
\hline 1. & & 111 & & 4444 & \\
\hline & & & & 3333 & \\
\hline 2111 & & 3333 & $\begin{array}{l}\frac{5}{74}=0.068 \\
\text { Age } 55\end{array}$ & 3332 & \\
\hline 1111 & & 4444 & $\frac{12}{75}=$ & 4444 & \\
\hline 222 & $\frac{8}{107}$ & 22 & $\frac{7}{75}=0$ & 3333 & $\frac{22}{231}=0.095$ \\
\hline 3332 & $\frac{5}{107}=0.047$ & 3232 & $\frac{3}{75}=0.053$ & 2222 & $\frac{18}{231}=0.078$ \\
\hline
\end{tabular}

See footnote 11 for details. 
Table 6: Sequences, Women

\begin{tabular}{|c|c|c|c|c|c|}
\hline \multicolumn{4}{|r|}{ HS Grad } & & HS \\
\hline \multicolumn{6}{|c|}{ Age 25} \\
\hline 1111 & $\frac{38}{215}=0.177$ & 1111 & $\frac{27}{205}=0.131$ & 2222 & $\frac{37}{354}=0.104$ \\
\hline 2222 & & 2222 & $\frac{24}{205}=0.117$ & 1111 & 0.068 \\
\hline 2232 & \multicolumn{4}{|c|}{ Age 30} & $\frac{23}{354}=$ \\
\hline 1111 & $\frac{74}{354}=0.209$ & 1111 & $\frac{31}{266}=0.117$ & 2222 & $\frac{51}{514}=0.099$ \\
\hline 2222 & $\frac{36}{354}=$ & 2222 & $\frac{22}{266}=0$ & 3333 & 070 \\
\hline 2212 & $\frac{12}{354}=0$ & 3333 & $\frac{11}{266}=0$ & 1111 & $\frac{27}{514}$ \\
\hline \multicolumn{6}{|c|}{ Age 35} \\
\hline 1111 & $\frac{83}{381}=0.218$ & 1111 & $\frac{33}{236}=0.140$ & 2222 & $\frac{42}{494}=0.085$ \\
\hline 2222 & $\frac{43}{381}=$ & 2222 & $\frac{25}{236}=0.106$ & 3333 & 085 \\
\hline 1112 & $\frac{16}{381}=0.042$ & 3333 & $\frac{16}{236}=0.068$ & 1111 & .057 \\
\hline \multicolumn{6}{|c|}{ Age 40} \\
\hline 1111 & $\frac{60}{295}=0.203$ & 1111 & $\frac{25}{197}=0.127$ & 2222 & $\frac{38}{413}=0.092$ \\
\hline 222 & $\frac{27}{295}=$ & 2222 & $\frac{16}{197}=$ & 1111 & 068 \\
\hline 3333 & $\frac{13}{295}=0.044$ & 3333 & $\frac{8}{197}=0.041$ & 3333 & $\frac{28}{413}=0.068$ \\
\hline \multicolumn{6}{|c|}{ Age 45} \\
\hline 1111 & $\frac{30}{188}=0.160$ & 2222 & $\frac{14}{124}=0.113$ & 4444 & $\frac{26}{323}=0.080$ \\
\hline 2222 & $\frac{17}{188}=$ & 1111 & $\frac{11}{124}=0.089$ & 1111 & 0.068 \\
\hline 3333 & $\frac{11}{188}=0.059$ & 4444 & $\begin{array}{l}\frac{8}{124}=0.065 \\
\text { Age } 50\end{array}$ & 2222 & $\frac{21}{323}=0.065$ \\
\hline 3333 & $\frac{11}{88}=0.125$ & 1111 & $\frac{12}{85}=0.141$ & 3333 & $\frac{35}{302}=$ \\
\hline & & 2222 & $\frac{6}{85}=0$. & 4444 & \\
\hline 1111 & $\frac{7}{88}=$ & 4444 & $\frac{5}{85}=0.059$ & 2222 & $\frac{21}{302}=0.070$ \\
\hline \multicolumn{6}{|c|}{ Age 55} \\
\hline 2222 & $\frac{13}{77}=$ & 3323 & $\frac{6}{69}=0.087$ & 4444 & $\frac{47}{309}=0.152$ \\
\hline 1111 & $\frac{8}{77}=$ & 1111 & $\frac{5}{69}=0.072$ & 3333 & $\frac{36}{309}=0.117$ \\
\hline 4444 & $\frac{6}{77}=0.078$ & 2222 & $\frac{5}{69}=0.072$ & 2222 & $\frac{\frac{22}{309}}{309}=0.071$ \\
\hline
\end{tabular}

See footnote 11 for details. 


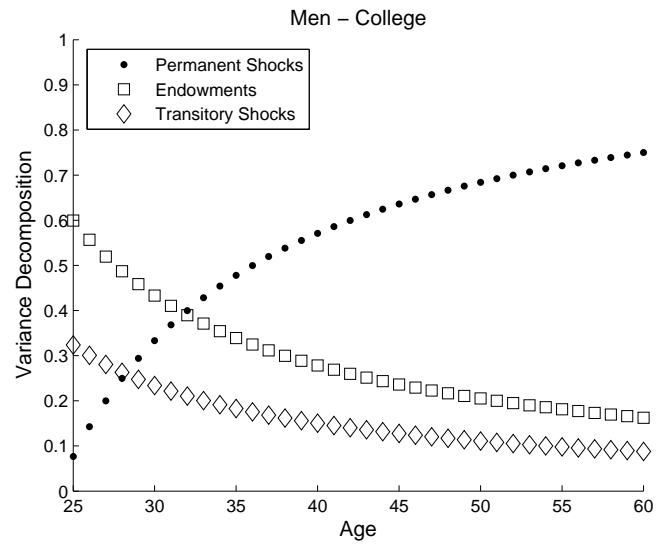

Figure 1

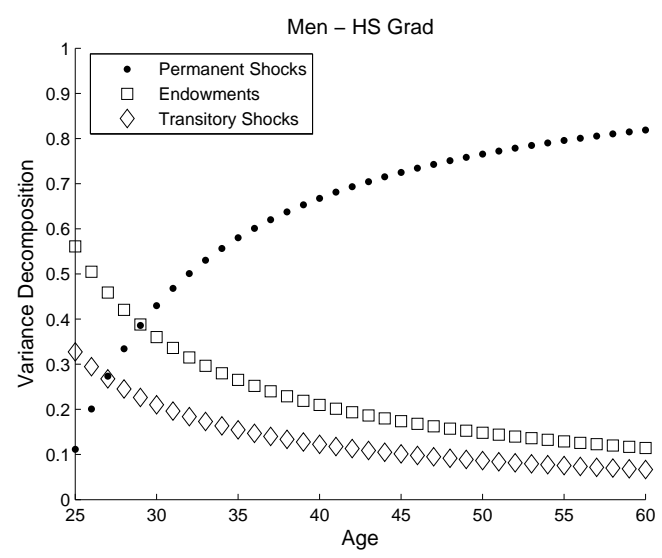

Figure 2

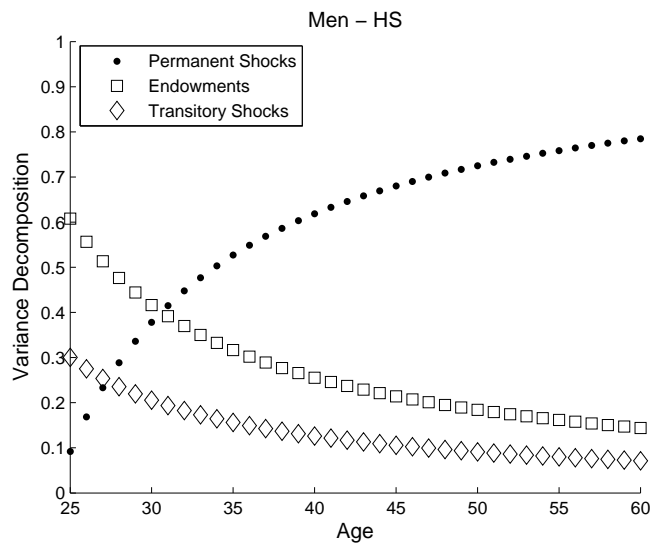

Figure 3

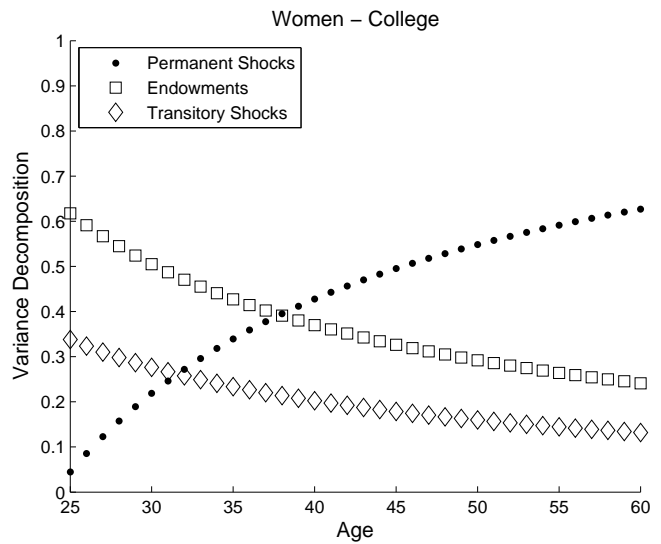

Figure 4

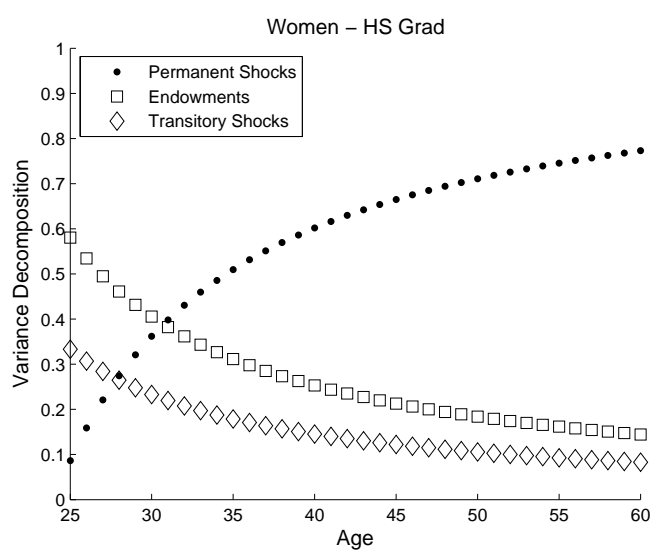

Figure 5

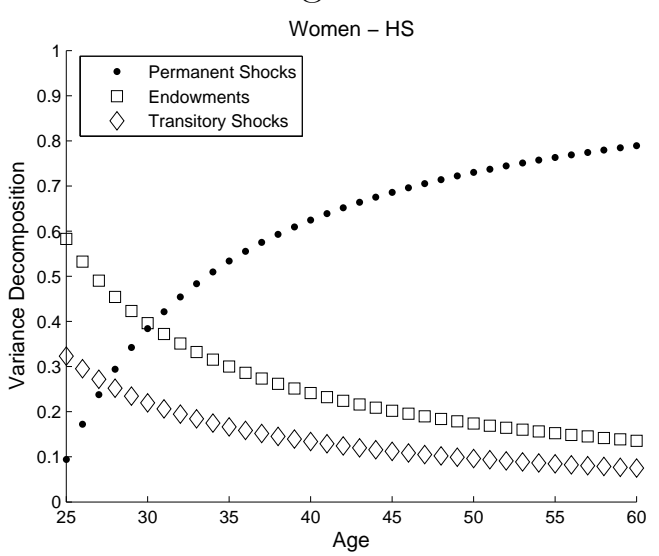

Figure 6 